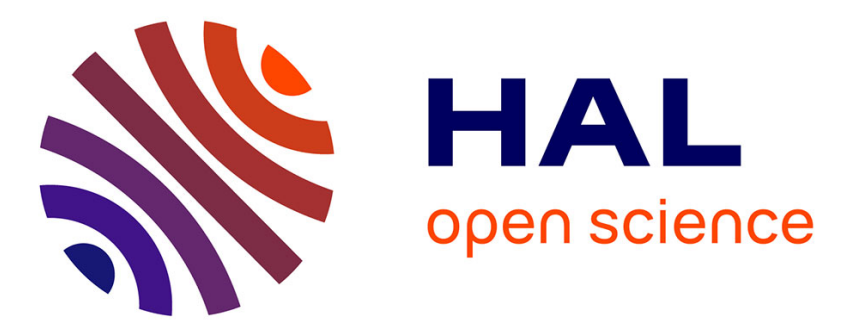

\title{
Low-complexity distributed fault detection for wireless sensor networks
}

Wenjie Li, Francesca Bassi, Davide Dardari, Michel Kieffer, Gianni Pasolini

\section{To cite this version:}

Wenjie Li, Francesca Bassi, Davide Dardari, Michel Kieffer, Gianni Pasolini. Low-complexity distributed fault detection for wireless sensor networks. IEEE International Conference on Communication, Jun 2015, London, United Kingdom. pp.6712 - 6718, 10.1109/ICC.2015.7249395 . hal-01260552

\section{HAL Id: hal-01260552 \\ https://hal.science/hal-01260552}

Submitted on 22 Jan 2016

HAL is a multi-disciplinary open access archive for the deposit and dissemination of scientific research documents, whether they are published or not. The documents may come from teaching and research institutions in France or abroad, or from public or private research centers.
L'archive ouverte pluridisciplinaire HAL, est destinée au dépôt et à la diffusion de documents scientifiques de niveau recherche, publiés ou non, émanant des établissements d'enseignement et de recherche français ou étrangers, des laboratoires publics ou privés.

\section{다(1)(2)}

Distributed under a Creative Commons Attribution - ShareAlikel 4.0 International 


\title{
Low-Complexity Distributed Fault Detection for Wireless Sensor Networks
}

\author{
Wenjie Li* ${ }^{*}$, Francesca Bassi*†, Davide Dardari ${ }^{\ddagger}$, Michel Kieffer*§ and Gianni Pasolini ${ }^{\ddagger}$ \\ *Laboratoire des Signaux et Systèmes (L2S, UMR CNRS 8506) CNRS-CentraleSupelec-Université Paris-Sud \\ 3, rue Joliot Curie 91192 Gif-sur-Yvette, France \\ ${ }^{\dagger}$ ESME-Sudria, 94200 Ivry-sur-Seine, France \\ $\ddagger$ CNIT, DEI, University of Bologna, Italy. \\ $\S$ LTCI Telecom ParisTech, 75013 Paris, France \\ ๆInstitut Universitaire de France, 75005 Paris, France
}

\begin{abstract}
To guarantee its integrity, a wireless sensor network needs to efficiently detect faulty nodes producing erroneous measurements. This paper proposes a fully distributed fault detection algorithm. A node first collects the measurements of its neighborhood, processes them to decide whether they contain outliers, and broadcasts the result. Then, it decides autonomously about its functioning status. The detection algorithm is proposed in two variants, depending on the proportion of faulty nodes in the network. A theoretical analysis of the probability of error and of the convergence of the algorithm is provided. The tradeoff between false alarm probability and detection probability is characterized using simulation. ${ }^{1}$
\end{abstract}

\section{INTRODUCTION}

A wireless sensor network (WSN) consists of many randomly deployed, autonomous devices, capable of acquiring physical measurements, of processing them, and of communicating over wireless channels. The role of a WSN is to evaluate some function of the whole set of measurements to accomplish a predefined task (e.g., monitoring object detection and tracking) [1].

Outliers are defined as abnormal measurements which cannot be justified by the mere effects of sensing noise. They may be informative because associated to the insurgence of some critical event (e.g., a forest fire), or caused by some sensor impairment. Outliers exhibit spatial and/or temporal correlation when indicating some event of interest (when the forest fire occurs, an entire region should have significantly higher temperature) [2], while can be assumed as independent if originated by defective sensors. Since the presence of uninformative outliers in the set of measurements may seriously disrupt the functionality of the system, the detection of the defective sensors is very important.

Many distributed fault detection (DFD) algorithms have already been proposed in the literature. The basic assumption is that all the nodes take noisy measurements of the same physical quantity. The algorithm devised in [3] allows each node to estimate its own functioning status by comparing the local measurement with the ones at neighboring nodes. In a first phase only a tendency status (good, faulty, likely good, or likely faulty) is determined. In the second phase the tendency status of neighbors are collected and associated to their measurements to obtain a more reliable assessment. In

\footnotetext{
${ }^{1}$ This work has been partly supported by the NEWCOM\# NoE
}

[4] the local test is based on the comparison between the local measurement and the median of the measurements of the neighbors. Iterative algorithms are proposed in [5], [6], where the weighted-median and the weighted average criterion are considered, respectively. In both cases the local test weights the measurements of the neighbors by the confidence level obtained from the previous detection round, under the assumption of permanent node failure. Intermittent faults in sensing and in communication are considered in [7], which proposes an adaptive DFD algorithm with a decision threshold adjusted at each round.

In this work we propose a low complexity DFD algorithm for large WSNs. Our approach differs with respect to classical solutions in the fact that the comparison of the local and neighboring measurements in the first phase of the algorithm only determines whether an outlier is present in the measurement set. This is usually an easier task than attempting to identify it. Consider, for example, three sensors measuring some constant temperature, e.g., with actual value $t=20^{\circ} \mathrm{C}$. Suppose that a non-defective sensor has a bounded measurement error, e.g., \pm 1 degree. Assume that the local measurement of the first sensor is $t_{1}=19.5^{\circ} \mathrm{C}$, and that two other sensors provide $t_{2}=20.8^{\circ} \mathrm{C}$ and $t_{3}=18.2^{\circ} \mathrm{C}$, respectively. Supposing that there is no defective sensor, and taking into account the bounded measurement noise, one deduces that $t \in \boldsymbol{t}_{1}=\left[t_{1}-1, t_{1}+1\right]=[18.5,20.5], t \in \boldsymbol{t}_{2}=[19.8,21.8]$, and $t \in \boldsymbol{t}_{3}=[17.2,19.2]$. Since $\boldsymbol{t}_{1} \cap \boldsymbol{t}_{2} \cap \boldsymbol{t}_{3}=\emptyset$, there exists at least one outlier. However, determining which sensor produces the outlier is difficult as $t_{1} \cap t_{2} \neq \emptyset$ and $t_{1} \cap t_{3} \neq \emptyset$. This type of result is reminiscent of group testing (GT) [8], a statistical technique which allows to identify a small number of defective items within a large population, with only a limited number of tests. A DFD algorithm based on centralized GT is proposed in [9], under the assumption of vanishing ratio of defective sensors. The centralized approach suffers a significant drawback in the communication overhead necessary for node indexing and for the dissemination of the test results from the cluster heads to the rest of the WSN.

In this paper we propose an algorithm able to overcome the mentioned problems. The algorithm works in two stages: measurement testing and fault detection. In the first stage each node collects measurements from its neighborhood, performs a local test to determine whether outliers are present among them, and broadcasts the result. Such tests are easily available, 
e.g., in the context of bounded-error estimation [10], [11]. Fault detection is performed in the second stage, on the basis of the outcomes of the measurement tests performed in the neighborhood. The robustness of the decision strategy is measured in terms of detection probability $\left(P_{\mathrm{D}}\right)$ and of false alarm probability $\left(P_{\mathrm{FA}}\right)$, defined, respectively, as the probability of defective sensors to be correctly detected, and the probability of non-defective sensors to be wrongly diagnosed as defective. The algorithm is proposed in two variants, non-adaptive and adaptive, the second being better suited to WSNs with a large proportion of defective sensors.

\section{Notations And System Model}

\section{A. Network model}

Consider an infinite plane where sensor nodes are uniformly and independently deployed, with spatial density $\rho$, according to a $2 \mathrm{D}$ Poisson point process (PPP) [12]. Let $\mathcal{S}$ denote the set of sensor nodes. A sensor is defective if it produces measurement outliers, i.e., measurement corrupted by noise samples whose characteristics differ significantly from those of non-defective sensors. An example of outlier models are introduced in Section IV-A. The sets of defective and nondefective (good) sensors are denoted by $\mathcal{D}$ and $\mathcal{G}$ respectively, with $\mathcal{D} \cup \mathcal{G}=\mathcal{S}$. The densities of defective and non-defective sensors are $\rho_{\mathrm{d}}$ and $\rho_{\mathrm{g}}$, with $\rho=\rho_{\mathrm{d}}+\rho_{\mathrm{g}}$. Let $\theta_{i}$ denote the status (defective or non-defective) of an arbitrary sensor $i \in \mathcal{S}$, i.e., $\theta_{i}=1$ if $i \in \mathcal{D}$ and $\theta_{i}=0$ if $i \in \mathcal{G}$. In this work, it is assumed that $\theta_{i}$ remains constant during the time interval in which the DFD is performed.

Assume that any pair of nodes $(i, j)$ can communicate if and only if $r_{i, j} \leq R_{0}$, where $r_{i, j}$ represents their physical distance and $R_{0}$ is the communication range, dependent on the transmission power, transmitter and receiver characteristics, and on the propagation scenario. Define $\mathcal{N}_{i}=$ $\left\{j \in \mathcal{S} \mid 0<r_{i, j} \leq R_{0}\right\}$ as the set of the neighbors of the node $i$. Let $\mathcal{U}_{i}=\mathcal{N}_{i} \cup\{i\}$. The probability of having $n$ sensors in $\mathcal{N}_{i}$ is [12]

$$
\operatorname{Pr}\left\{\left|\mathcal{N}_{i}\right|=n\right\}=\frac{\mu^{n}}{n !} \exp (-\mu),
$$

where $\mu=\rho \pi R_{0}^{2}$ is the average number of sensors in the neighborhood of sensor $i$. Similarly, one has $\mu_{\mathrm{g}}=\rho_{\mathrm{g}} \pi R_{0}^{2}$ and $\mu_{\mathrm{d}}=\rho_{\mathrm{d}} \pi R_{0}^{2}$. The probability of having $n_{\mathrm{d}}$ defective sensors and $n_{\mathrm{g}}=n-n_{\mathrm{d}}$ non-defective sensors in $\mathcal{N}_{i}$ is [12]

$$
\operatorname{Pr}\left\{\left|\mathcal{N}_{i} \cap \mathcal{G}\right|=n_{\mathrm{g}},\left|\mathcal{N}_{i} \cap \mathcal{D}\right|=n_{\mathrm{d}}\right\}=\frac{\mu_{\mathrm{d}}^{n_{\mathrm{d}}} \mu_{\mathrm{g}}^{n_{\mathrm{g}}}}{n_{\mathrm{d}} ! n_{\mathrm{g}} !} \exp (-\mu),
$$

notice that $\mu=\mu_{\mathrm{g}}+\mu_{\mathrm{d}}$.

\section{B. Outlier detection test}

Consider a generic set of sensors $\mathcal{A} \subseteq \mathcal{S}$ and define

$$
\varphi(\mathcal{A})= \begin{cases}1, & \text { if } \mathcal{A} \cap \mathcal{D} \neq \emptyset \\ 0, & \text { otherwise }\end{cases}
$$

as the indicator function for the presence of defective sensors in $\mathcal{A}$. At a given time instant, let the random variable $M_{i} \in \mathbb{R}$ denote the measurement provided by sensor $i \in \mathcal{A}$. The measurements of all sensors in $\mathcal{A}$ are gathered in $\mathbf{M}_{\mathcal{A}}=$
$\left[M_{i}\right]_{i \in \mathcal{A}} \in \mathbb{R}^{|\mathcal{A}|}$. The physical quantities measured by the sensors may be spatially correlated. The measurement noises produced by different sensors are assumed as uncorrelated. For a given realization $\mathbf{m}_{\mathcal{A}} \in \mathbb{R}^{|\mathcal{A}|}$ of $\mathbf{M}_{\mathcal{A}}$, denote $T\left(\mathbf{m}_{\mathcal{A}}\right)$ the outcome of some local outlier detection test

$$
T\left(\mathbf{m}_{\mathcal{A}}\right)= \begin{cases}1, & \text { if at least an outlier is detected in } \mathbf{m}_{\mathcal{A}}, \\ 0, & \text { otherwise. }\end{cases}
$$

Even if $\varphi(\mathcal{A})=1$, i.e., at least one defective sensor belongs to $\mathcal{A}$, the noise characteristics of the defective sensors may produce some realizations $\mathbf{m}_{\mathcal{A}}$ that do not allow the detection of the outliers. Thus, one introduces the probability of outlier detection

$$
q_{\mathrm{D}}\left(\mathbf{M}_{\mathcal{A}}\right)=\operatorname{Pr}\left\{T\left(\mathbf{M}_{\mathcal{A}}\right)=1 \mid \varphi(\mathcal{A})=1\right\} .
$$

Similarly, one considers the probability of false alarm

$$
q_{\mathrm{FA}}\left(\mathbf{M}_{\mathcal{A}}\right)=\operatorname{Pr}\left\{T\left(\mathbf{M}_{\mathcal{A}}\right)=1 \mid \varphi(\mathcal{A})=0\right\} .
$$

Specific properties of $q_{\mathrm{FA}}$ and $q_{\mathrm{D}}$ are provided in Section IV. They depend on the considered fault model.

\section{Distributed FAult Detection Algorithm}

This section proposes two variants of a DFD algorithm (non-adaptive and adaptive) to estimate the status of each sensor using some local outlier detection test $T$. The variant to be chosen depends on the defective sensor ratio $\mu_{\mathrm{d}} / \mu$.

\section{A. Non-adaptive DFD}

The non-adaptive version of the proposed DFD algorithm consists of two successive phases, repeated for $L$ rounds, followed by a final phase, whose outcome is, at each node $i$, an estimate $\widehat{\theta}_{i}$ of $\theta_{i}$.

\section{Algorithm 1 Non-adaptive DFD \\ 1) Initialize $\widehat{\theta}_{i}^{(0)}=0$ and $z_{i}^{(0)}=0$ for all $i \in \mathcal{S}$. \\ 2) For each round $1 \leq \ell \leq L$ :}

- Phase I: each sensor $i$ broadcasts a packet containing its local measurement $m_{i}^{(\ell)}$, receives the measurements produced by the sensors in $\mathcal{N}_{i}$ and performs the test (4) with outcome

$$
y_{i}^{(\ell)}=T\left(\mathbf{m}_{\mathcal{U}_{i}}^{(\ell)}\right)
$$

- $\quad$ Phase II: each sensor broadcasts $y_{i}^{(\ell)}$ generated in Phase I and updates $z_{i}^{(\ell)}$ as follows

$$
z_{i}^{(\ell)}=z_{i}^{(\ell-1)}+\sum_{j \in \mathcal{U}_{i}} y_{j}^{(\ell)}
$$

to accumulate the number of neighbors that have a positive test result at Phase I up to round $\ell$.

3) After $L$ rounds:

- $\quad$ Phase III: each sensor $i$ estimates its status $\theta_{i}$

$$
\widehat{\theta}_{i}=\left\{\begin{array}{lll}
1 & \text { (defective) } & \text { if } \frac{z_{i}^{(L)}}{L\left|\mathcal{U}_{i}\right|}>\gamma, \\
0 & \text { (non defective) } & \text { otherwise }
\end{array}\right.
$$

where $\gamma$ is some threshold. 


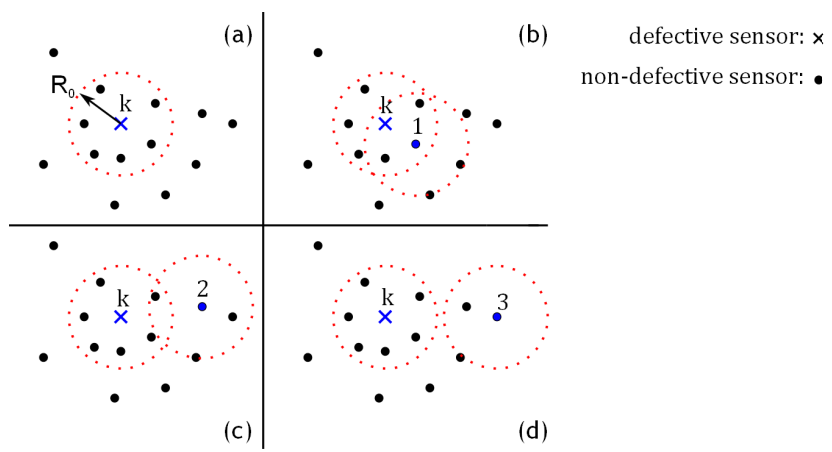

Fig. 1. Example where $k$ is the only defective sensor, $(a)$ shows that $z_{k}^{1}=$ $\left|\mathcal{U}_{k}\right|=7$. In $(b)$, sensor 1 belongs to $\mathcal{U}_{k}$ and $z_{1}^{1}=4$. In $(c)$, sensor 2 is not in $\mathcal{U}_{k}$ but $r_{2, k} \leq 2 R_{0}$, which results in $z_{2}^{1}=1$. In $(d)$, sensor 3 has a distance $r_{2, k}>2 R_{0}$, so $z_{3}^{1}=0$.

The decision (9) in Phase III can result in both false alarm (with probability $P_{\mathrm{FA}}$ ) and non-detection (with probability $1-P_{\mathrm{D}}$ ). The values of $\gamma$ and $L$ determine the trade-off between $P_{\mathrm{D}}$ and $P_{\mathrm{FA}}$. They have to be adjusted to meet the targeted performance. When $\gamma$ is too large, $P_{\mathrm{D}}$ may be low, with a significant number of defective sensors diagnosed as good. On the other hand, $P_{\mathrm{FA}}$ may be high for small values of $\gamma$. Increasing $L$ provides a better averaging effect in (9), which reduces the variance of $\widehat{\theta}_{i}$. However, $L$ cannot be taken too large, to preserve the assumption of constant $\theta_{i}$ s during $L$ rounds.

Example 1. Figure 1 depicts a toy example. Let $k$ be the only defective sensor. Assume that both $q_{\mathrm{FA}}$ defined in (6) and $q_{\mathrm{ND}}$ defined in (5) are negligible: all the sensors in $\mathcal{U}_{k}$ successfully detect outliers. Moreover consider $L=1$ (unique round) and take $\gamma=0.7$. At the end of Phase II, $z_{k}^{(1)}=$ $\left|\mathcal{U}_{k}\right|=7, z_{1}^{(1)}=\left|\mathcal{U}_{k} \cap \mathcal{U}_{1}\right|=4, z_{2}^{(1)}=\left|\mathcal{U}_{k} \cap \mathcal{U}_{2}\right|=1$, and $z_{3}^{(1)}=\left|\mathcal{U}_{k} \cap \mathcal{U}_{3}\right|=0$. Since $z_{k}^{(1)} /\left|\mathcal{U}_{k}\right|=1>\gamma, z_{1}^{(1)} /\left|\mathcal{U}_{1}\right| \approx$ $0.67<\gamma, z_{2}^{(1)} /\left|\mathcal{U}_{2}\right|=0.25<\gamma$, and $z_{3}^{(1)} /\left|\mathcal{U}_{3}\right|=0<\gamma$, only sensor $k$ determines itself as defective, according to (9), while sensors 1, 2, and 3 diagnose themselves as non-defective.

As it will be discussed in Section VI, the non-adaptive DFD performs well when $\mu_{\mathrm{d}} \ll \mu_{\mathrm{g}}$. However, $P_{\mathrm{FA}}$ rapidly increases with $\mu_{\mathrm{d}} / \mu$. In the critical situation, where every non-defective sensor has at least one defective sensor in its neighborhood, for all sensors $i \in \mathcal{S}$ the test outcome is $y_{i}^{(\ell)}=1$ with a high probability. As a consequence, non-defective sensors are frequently diagnosed as defective.

\section{B. Adaptive DFD}

To improve the non-adaptive DFD algorithm for increasing $\mu_{\mathrm{d}} / \mu$, an adaptive version is described in Algorithm 2. In the adaptive algorithm, sensors are allowed to take temporary decisions about their status. At each of the first $L_{1}$ rounds of the algorithm, measurements are exchanged with neighbors and a temporary decision $\widehat{\theta}_{i}^{(\ell)}$ is taken. The decision at round $\ell-1$ affects the set of sensors from which sensor $i$ gets information at round $\ell$, i.e., $\widehat{\mathcal{U}}_{i}^{(\ell)}=\left\{j \in \mathcal{U}_{i}\right.$ s.t. $\left.\widehat{\theta}_{j}^{(\ell-1)}=0\right\}$. This first part of the algorithm allows to discover most of the defective sensors, while keeping $P_{\mathrm{FA}}$ small.

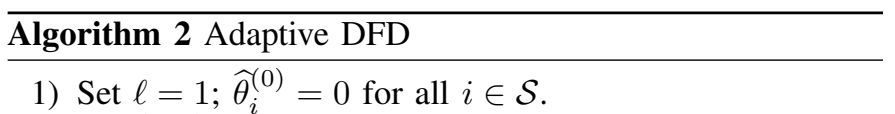

2) If $\widehat{\theta}_{i}^{(\ell-1)}=0$, sensor $i$ broadcasts its measurement, calculates the $\operatorname{sum} z_{i}^{(\ell)}=\sum_{j \in \widehat{\mathcal{U}}_{i}^{(\ell)}} y_{j}^{(\ell)}$, and performs the decision

$$
\widehat{\theta}_{i}^{(\ell)}= \begin{cases}1, & \text { if } z_{i}^{(\ell)} /\left|\widehat{\mathcal{U}}_{i}^{(\ell)}\right|>\gamma_{1}, \\ 0, & \text { otherwise. }\end{cases}
$$

3) If $\widehat{\theta}_{i}^{(\ell-1)}=1$, sensor $i$ is silent, i.e., it does not broadcast its measurement and performs the decision $\widehat{\theta}_{i}^{(\ell)}=y_{i}^{(\ell)}$.

4) $\ell=\ell+1$. If $\ell \leq L_{1}$, go to 2 .

5) After round $L_{1}$ :

- $\quad$ each sensor $i$ such that $\widehat{\theta}_{i}^{\left(L_{1}\right)}=0$ sets $z_{i}^{\left(L_{1}\right)}=0$.

- $\quad$ each sensor $i$ such that $\widehat{\theta}_{i}^{\left(L_{1}\right)}=1$ is determined as defective. It stops broadcasting its measurements. It does not participate in the non-adaptive DFD during the following rounds.

6) Non-adaptive DFD is performed during $L_{2}$ rounds with threshold $\gamma_{2}$.

At the beginning of the algorithm, a large amount of nondefective sensors may be diagnosed as defective. In Step 3, sensors become silent and stop broadcasting their measurements if they are temporarily determined as defective. Step 4 gives a chance to revive to the non-defective sensors erroneously deemed defective (the $P_{\mathrm{FA}}$ is high at the beginning): if $\widehat{\theta}_{i}^{(\ell-1)}=1$, the decision is made only based on the local test of sensor $i$. In few rounds, $P_{\mathrm{FA}}$ can thus be reduced to an acceptable level. The following $L_{2}$ rounds help to increase $P_{\mathrm{D}}$, as shown in Section VI.

\section{Outlier Detection Test}

This section presents an example of outlier model and a possible detection test.

\section{A. Outlier model}

Assume that each sensor $i$ gets a noisy observation of some scalar physical quantity $\phi$

$$
m_{i}=\phi+w_{i}, \quad \forall i \in \mathcal{S} .
$$

The components $w_{i}$ of the measurement noise in (11) are assumed to be realizations of independent Gaussian random variables $W_{i}$ with the same standard deviation $\sigma$. For good sensors (i.e., $i \in \mathcal{G}$ ), the measurement noise is assumed zeromean. For the defective sensors, $W_{i}$ has a random bias $E_{i}$ uniformly distributed over $[-3 \eta \sigma, 3 \eta \sigma]$, where $\eta>1$. With this setting, each defective sensor has a different value of bias.

\section{B. Outlier detection test}

Consider the interval $\left[m_{i}\right]=\left[m_{i}-\Delta, m_{i}+\Delta\right]$ of width $2 \Delta$ centered around the measurement $m_{i}$. If $i \in \mathcal{G}$, one has $\operatorname{Pr}\left\{\phi \in\left[m_{i}\right]\right\}=1-\operatorname{erfc}\left(\frac{\Delta}{\sqrt{2} \sigma}\right)$. If $i \in \mathcal{D}$, similarly, one evaluates the probability that $\phi \notin\left[m_{i}\right]$ as a function of $\sigma, \Delta$, and $\eta$, when the fault model is that of Section IV-A. 
Consider now the intersection of all $\left[m_{i}\right], \forall i \in \mathcal{A}$ :

$$
\left[\widehat{\phi}\left(\mathbf{m}_{\mathcal{A}}\right)\right]=\bigcap_{i \in \mathcal{A}}\left[m_{i}\right] .
$$

With and without presence of outliers, one is able to evaluate $\operatorname{Pr}\{[\hat{\phi}]=\emptyset\}$ as a function of $\sigma, \eta$, and $\Delta$. These two quantities may be used to define a low-complexity outlier detection test

$$
T\left(\mathbf{m}_{\mathcal{A}}\right)= \begin{cases}1, & \text { if }\left[\widehat{\phi}\left(\mathbf{m}_{\mathcal{A}}\right)\right]=\emptyset \\ 0, & \text { else. }\end{cases}
$$

With this test, $q_{\mathrm{FA}}$ and $q_{\mathrm{D}}$ defined in (6) and (5) only depend on $n_{\mathrm{g}}$ and $n_{\mathrm{d}}$, where $n_{\mathrm{g}}=|\mathcal{A} \cap \mathcal{G}|$ and $n_{\mathrm{d}}=|\mathcal{A} \cap \mathcal{D}|$. As $\left[\widehat{\phi}\left(\mathbf{m}_{\mathcal{A}} \cup m_{j}\right)\right] \subseteq\left[\widehat{\phi}\left(\mathbf{m}_{\mathcal{A}}\right)\right], T\left(\mathbf{m}_{\mathcal{A}}\right)$ is more probable to get close to 1 as the number of measurements increase. Therefore, $q_{\mathrm{FA}}\left(n_{\mathrm{g}}\right)$ is an increasing function of $n_{\mathrm{g}}$, and $q_{\mathrm{D}}\left(n_{\mathrm{g}}, n_{\mathrm{d}}\right)$ is an increasing function of both $n_{\mathrm{g}}$ and $n_{\mathrm{d}}$.

\section{Theoretical Analysis}

In this section we characterize analytically the probabilities of fault detection $P_{\mathrm{D}}$ and of false alarm $P_{\mathrm{FA}}$ of the proposed DFD algorithm, when the decision is taken after one single round of the non-adaptive algorithm, i.e., after a single collection of measurements from neighbors. Conditions for the convergence of the adaptive algorithm are also evaluated.

\section{A. $P_{D}$ and $P_{F A}$ for a single round of the non-adaptive DFD algorithm}

\section{Consider the event}

$$
\mathcal{E}_{i, \mathrm{D}}(n)=\left\{\sum_{j \in \mathcal{U}_{i}} Y_{j}>\gamma(n+1)\left|\theta_{i}=1,\right| \mathcal{N}_{i} \mid=n\right\},
$$

representing, according to (9), the situation where a defective sensor $i$ is detected as defective considering data coming from $n$ neighbors. The detection probability of a defective sensor can be hence expressed as

$$
P_{\mathrm{D}}=\sum_{n=0}^{\infty} \operatorname{Pr}\left\{\mathcal{E}_{i, \mathrm{D}}(n)\right\} \operatorname{Pr}\left\{\left|\mathcal{N}_{i}\right|=n\right\}
$$

As sensors cannot perform outlier detection when they have no neighbors, $\operatorname{Pr}\left\{\mathcal{E}_{i, \mathrm{D}}(0)\right\}=1$.

Similarly, consider a non-defective sensor $i$, the conditional false alarm event is

$$
\mathcal{E}_{i, \mathrm{FA}}(n)=\left\{\sum_{j \in \mathcal{U}_{i}} Y_{j}>\gamma(n+1)\left|\theta_{i}=0,\right| \mathcal{N}_{i} \mid=n\right\} .
$$

The probability of false alarm is given by

$$
P_{\mathrm{FA}}=\sum_{n=0}^{\infty} \operatorname{Pr}\left\{\mathcal{E}_{i, \mathrm{FA}}(n)\right\} \operatorname{Pr}\left\{\left|\mathcal{N}_{i}\right|=n\right\} .
$$

Lemma 1. Both $P_{D}$ and $P_{F A}$ are decreasing functions of $\gamma$.

Proof: The monotonicity of $P_{\mathrm{D}}$ and $P_{\mathrm{FA}}$ with respect to $\gamma$ is obvious, since if $\gamma_{1}>\gamma_{2}, \sum_{j \in \mathcal{U}_{i}} Y_{j}>\gamma_{1}(n+1)$ implies $\sum_{j \in \mathcal{U}_{i}} Y_{j}>\gamma_{2}(n+1)$.
Consider now the event

$$
\mathcal{Y}_{j, \mathrm{D}}(n)=\left\{Y_{j}=1\left|j \in \mathcal{U}_{i}, \theta_{i}=1,\right| \mathcal{N}_{i} \mid=n\right\},
$$

with $n>0$ and $j \in \mathcal{U}_{i}$. For any $j^{\prime} \in \mathcal{U}_{i}$ such that $j \neq j^{\prime}$, $\mathcal{Y}_{j, \mathrm{D}}(n)$ and $\mathcal{Y}_{j^{\prime}, \mathrm{D}}(n)$ are dependent. Their dependence comes from the fact that in general $\mathcal{N}_{j} \cap \mathcal{N}_{j^{\prime}} \neq \emptyset$. The probability mass function (pmf) of $\sum_{j \in \mathcal{U}_{i}} Y_{j}$, necessary to evaluate $P_{\mathrm{D}}$, is thus quite difficult to evaluate, since the dependency between the $Y_{j} \mathrm{~s}$ is not explicit. The exact form of $P_{\mathrm{D}}$ then becomes quite complicated, but we can make the following conjecture.

Conjecture 1. $P_{D}$ is an increasing function of $\mu_{d}$. Moreover, $\mu_{d} P_{D}$ is a convex function of $\mu_{d}$.

The decision concerning the status of sensor $i$ at the end of the DFD algorithm is based on the results of the local tests of all the sensors in $\mathcal{U}_{i}$. If the local test results are more likely to be 1 , the final decision is reasonably more probable to be 1 . Thus, one conjectures that the monotonicity of $P_{\mathrm{D}}$ with respect to $\mu_{\mathrm{d}}$ is inherited by the monotonicity of $\operatorname{Pr}\left\{Y_{i}=1 \mid \theta_{i}=1\right\}$ and of $\operatorname{Pr}\left\{Y_{i}=1 \mid \theta_{i}=0\right\}$ with respect to $\mu_{\mathrm{d}}$. Define the function

$$
h\left(n_{\mathrm{g}}, n_{\mathrm{d}}\right)= \begin{cases}q_{\mathrm{FA}}\left(n_{\mathrm{g}}\right), & \text { if } n_{\mathrm{d}}=0, \\ 1-q_{\mathrm{ND}}\left(n_{\mathrm{g}}, n_{\mathrm{d}}\right), & \text { if } n_{\mathrm{d}} \neq 0,\end{cases}
$$

representing the probability of having $Y_{i}=1$ in a local test, knowing that $\left|\mathcal{G} \cap \mathcal{U}_{i}\right|=n_{\mathrm{g}},\left|\mathcal{D} \cap \mathcal{U}_{i}\right|=n_{\mathrm{d}}$. Thus, one may introduce

$$
\begin{aligned}
f_{1}\left(\mu_{\mathrm{g}}, \mu_{\mathrm{d}}\right) & =\operatorname{Pr}\left\{Y_{i}=1 \mid \theta_{i}=0\right\} \\
& =\sum_{n_{\mathrm{g}}=0}^{\infty} \sum_{n_{\mathrm{d}}=0}^{\infty} h\left(n_{\mathrm{g}}+1, n_{\mathrm{d}}\right) \frac{\mu_{\mathrm{d}}^{n_{\mathrm{d}}} \mu_{\mathrm{g}}^{n_{\mathrm{g}}}}{n_{\mathrm{d}} ! n_{\mathrm{g}} !} \exp \left(-\mu_{\mathrm{d}}-\mu_{\mathrm{g}}\right),
\end{aligned}
$$

and

$$
\begin{aligned}
f_{2}\left(\mu_{\mathrm{g}}, \mu_{\mathrm{d}}\right) & =\operatorname{Pr}\left\{Y_{i}=1 \mid \theta_{i}=1\right\} \\
& =\sum_{n_{\mathrm{g}}=0}^{\infty} \sum_{n_{\mathrm{d}}=0}^{\infty} h\left(n_{\mathrm{g}}, n_{\mathrm{d}}+1\right) \frac{\mu_{\mathrm{d}}^{n_{\mathrm{d}}} \mu_{\mathrm{g}}^{n_{\mathrm{g}}}}{n_{\mathrm{d}} ! n_{\mathrm{g}} !} \exp \left(-\mu_{\mathrm{d}}-\mu_{\mathrm{g}}\right) .
\end{aligned}
$$

One has

$$
\begin{aligned}
\frac{\partial f_{1}}{\partial \mu_{\mathrm{g}}}=\sum_{n_{\mathrm{g}}=0}^{\infty} \sum_{n_{\mathrm{d}}=0}^{\infty} \frac{\mu_{\mathrm{d}}^{n_{\mathrm{d}}} \mu_{\mathrm{g}}^{n_{\mathrm{g}}}}{n_{\mathrm{d}} ! n_{\mathrm{g}} !} \exp \left(-\mu_{\mathrm{d}}-\mu_{\mathrm{g}}\right) \\
\cdot\left(h\left(n_{\mathrm{g}}+2, n_{\mathrm{d}}\right)-h\left(n_{\mathrm{g}}+1, n_{\mathrm{d}}\right)\right)>0
\end{aligned}
$$

as $h\left(n_{\mathrm{g}}+2, n_{\mathrm{d}}\right)>h\left(n_{\mathrm{g}}+1, n_{\mathrm{d}}\right)$. Similarly, one has $\frac{\partial f_{1}}{\partial \mu_{\mathrm{d}}}>0$, $\frac{\partial f_{2}}{\partial \mu_{\mathrm{g}}}>0$, and $\frac{\partial f_{2}}{\partial \mu_{\mathrm{d}}}>0$. We can conjecture that $P_{\mathrm{D}}$ is an increasing function of $\mu_{\mathrm{d}}$ and $\mu_{\mathrm{d}} P_{\mathrm{D}}$ is a convex function of $\mu_{\mathrm{d}}$. These conjectures are verified experimentally in Section VI.

The monotonicity of $P_{\mathrm{FA}}$ with respect to $\mu_{\mathrm{g}}$ is more complicated to analyze qualitatively. As $\mu_{\mathrm{g}}$ increases, a good sensor that has a defective sensor as its neighbor is more probable to be diagnosed as defective. On the other hand, the probability of having one defective sensor as neighbor decreases with $\mu_{\mathrm{g}}$.

Conjecture 2. $P_{F A}$ is a decreasing function of $\mu_{g}$, while $\mu_{g} P_{F A}$ is an increasing and concave function of $\mu_{g}$. 

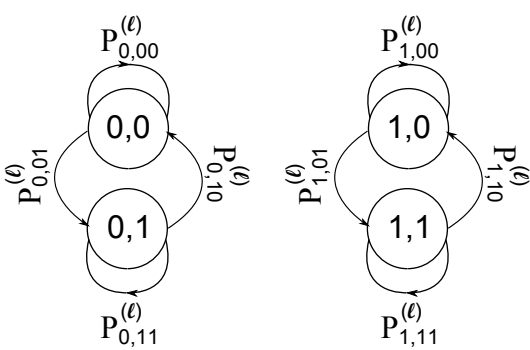

Fig. 2. Evolution with the rounds of the state of a sensor when applying the adaptive DFD, notice that both automatas do not evolve in parallel independently.

Again, Conjecture 2 is verified experimentally in Section VI.

\section{B. Convergence of the adaptive algorithm}

Let the pair $\left(\theta_{i}, \hat{\theta}_{i}\right)$ denote the state of sensor $i$, where $\theta_{i}$ is its actual status and $\hat{\theta}_{i}$ is its estimated status. Among the four possible states, $(0,0)$ and $(1,1)$ are states resulting from a correct decision, $(0,1)$ corresponds to a false alarm, and $(1,0)$ corresponds to a non-detection. Let $\mu_{\theta_{i}, \hat{\theta}_{i}}^{(\ell)}$ denote the density of sensors in the state $\left(\theta_{i}^{(\ell)}, \hat{\theta}_{i}^{(\ell)}\right)$ at round $\ell$. The aim of this section is to characterize the evolution of $\mu_{\theta_{i}, \hat{\theta}_{i}}^{(\ell)}$ to determine whether the adaptive algorithm converges to a steady state. The transition between states is described in Figure 2.

Before the first round of the adaptive algorithm, one has $\mu_{00}^{(0)}=\mu_{\mathrm{g}}, \mu_{10}^{(0)}=\mu_{\mathrm{d}}$, and $\mu_{01}^{(0)}=\mu_{11}^{(0)}=0$. Note that at any round $\ell, \mu_{00}^{(\ell)}+\mu_{01}^{(\ell)}=\mu_{\mathrm{g}}$ and $\mu_{10}^{(\ell)}+\mu_{11}^{(\ell)}=\mu_{\mathrm{d}}$. The transitions from round $\ell$ to round $\ell+1$ are illustrated in Figure 2. Since the actual status of a sensor is assumed constant during the rounds of the DFD algorithm, the only possible transitions are between states $(0,0)$ and $(0,1)$ and between states $(1,0)$ and $(1,1)$. The evolution of the densities between rounds $\ell$ and $\ell+1$ is given by

$$
\left[\begin{array}{c}
\mu_{00}^{(\ell+1)} \\
\mu_{01}^{(\ell+1)} \\
\mu_{10}^{(\ell+1)} \\
\mu_{11}^{(\ell+1)}
\end{array}\right]=\left[\begin{array}{cccc}
P_{0,00}^{(\ell)} & P_{0,10}^{(\ell)} & 0 & 0 \\
P_{0,01}^{(\ell)} & P_{0,11}^{(\ell)} & 0 & 0 \\
0 & 0 & P_{1,00}^{(\ell)} & P_{1,10}^{(\ell)} \\
0 & 0 & P_{1,01}^{(\ell)} & P_{1,11}^{(\ell)}
\end{array}\right]\left[\begin{array}{c}
\mu_{00}^{(\ell)} \\
\mu_{01}^{(\ell)} \\
\mu_{10}^{(\ell)} \\
\mu_{11}^{(\ell)}
\end{array}\right],
$$

with:

$P_{0,01}^{(\ell)}$ is the transition probability from $(0,0)$ to $(0,1)$. It is equal to $P_{\mathrm{FA}}$ in the first round. The sensors considered as defective in the previous round are silent, so $P_{\mathrm{FA}}$ is a function of $\mu_{00}^{(\ell)}$ and $\mu_{10}^{(\ell)}$, denoted as $P_{\mathrm{FA}}\left(\mu_{00}^{(\ell)}, \mu_{10}^{(\ell)}\right)$. Obviously, $P_{0,00}^{(\ell)}=1-P_{0,01}^{(\ell)}$.

$P_{0,10}^{(\ell)}$ is the transition probability from $(0,1)$ to $(0,0)$. Suppose that sensor $i$ is in the state $(0,1)$. Recall that in the proposed algorithm, sensor $i$ does not broadcast, but still collects measurements from its neighbors in state $(0,0)$ or $(1,0)$. The measurement of sensor $i$ is only considered in its own local test. From (20), one deduces that $P_{0,10}^{(\ell)}=1-f_{1}\left(\mu_{00}^{(\ell)}, \mu_{10}^{(\ell)}\right)$ and $P_{0,11}^{(\ell)}=f_{1}\left(\mu_{00}^{(\ell)}, \mu_{10}^{(\ell)}\right)$.
$P_{1,00}^{(\ell)}$ is the transition probability from $(1,0)$ to $(1,0)$. This is the probability of mis-detection of a defective sensor, i.e., $1-P_{\mathrm{D}}\left(\mu_{00}^{(\ell)}, \mu_{10}^{(\ell)}\right)$. Moreover $P_{1,01}^{(\ell)}=P_{\mathrm{D}}\left(\mu_{00}^{(\ell)}, \mu_{10}^{(\ell)}\right)$.

$P_{1,10}^{(\ell)}$ is the transition probability from $(1,1)$ to $(1,0)$, which is similar to $P_{0,10}^{(\ell)}$. One has $P_{1,10}^{(\ell)}=1-f_{2}\left(\mu_{00}^{(\ell)}, \mu_{10}^{(\ell)}\right)$ and $P_{1,11}^{(\ell)}=f_{2}\left(\mu_{00}^{(\ell)}, \mu_{10}^{(\ell)}\right)$.

From (23), one gets

$$
\begin{aligned}
\mu_{00}^{(\ell+1)} & =\mu_{00}^{(\ell)} P_{0,00}^{(\ell)}+\left(\mu_{\mathrm{g}}-\mu_{00}^{(\ell)}\right) P_{0,10}^{(\ell)} \\
& =\mu_{00}^{(\ell)}\left(f_{1}-P_{\mathrm{FA}}\right)+\mu_{\mathrm{g}}\left(1-f_{1}\right)
\end{aligned}
$$

and

$$
\begin{aligned}
\mu_{10}^{(\ell+1)} & =\mu_{10}^{(\ell)} P_{1,00}^{(\ell)}(\ell)+\left(\mu_{\mathrm{d}}-\mu_{10}^{(\ell)}\right) P_{1,10}^{(\ell)} \\
& =\mu_{10}^{(\ell)}\left(f_{2}-P_{\mathrm{D}}\right)+\mu_{\mathrm{d}}\left(1-f_{2}\right) .
\end{aligned}
$$

To lighten notations, the arguments $\mu_{00}^{(\ell)}$ and $\mu_{10}^{(\ell)}$ of $P_{\mathrm{FA}}, P_{\mathrm{D}}$, $f_{1}$, and $f_{2}$ have been omitted in (24), (25), and in what follows when there is no confusion.

Let $\mu_{00}^{*}$ and $\mu_{10}^{*}$ be the values at equilibrium of $\mu_{00}^{(\ell)}$ and $\mu_{10}^{(\ell)}$, respectively. From (24) and (25), one deduces that $\mu_{00}^{*}$ and $\mu_{10}^{*}$ should satisfy

$$
\mu_{\mathrm{g}}-\mu_{00}^{*}=\frac{\mu_{00}^{*} P_{\mathrm{FA}}\left(\mu_{00}^{*}, \mu_{10}^{*}\right)}{1-f_{1}\left(\mu_{00}^{*}, \mu_{10}^{*}\right)},
$$

and

$$
\mu_{\mathrm{d}}-\mu_{10}^{*}=\frac{\mu_{10}^{*} P_{\mathrm{D}}\left(\mu_{00}^{*}, \mu_{10}^{*}\right)}{1-f_{2}\left(\mu_{00}^{*}, \mu_{10}^{*}\right)} .
$$

Lemma 2. Assume that Conjectures 1 and 2 hold, then (26) and (27) admit a unique solution.

Proof: In the case of (27), when we study the left-hand side, $\mu_{\mathrm{g}}-\mu_{00}^{*}$ is a continuous and monotonically decreasing function of $\mu_{00}^{*}$ over $\left[0, \mu_{\mathrm{g}}\right]$, with

$$
\lim _{\mu_{00}^{*} \rightarrow 0} \mu_{\mathrm{g}}-\mu_{00}^{*}=\mu_{\mathrm{g}} \quad \text { and } \quad \lim _{\mu_{00}^{*} \rightarrow \mu_{\mathrm{g}}} \mu_{\mathrm{g}}-\mu_{00}^{*}=0 .
$$

We need now to study the behavior of the right hand side for a fixed value of $\mu_{10}^{*}$. As $f_{1}$ and $\mu_{00}^{*} P_{\mathrm{FA}}$ are increasing functions of $\mu_{00}^{*}$, obviously, $\mu_{00}^{*} P_{\mathrm{FA}} /\left(1-f_{1}\right)$ is an increasing function of $\mu_{00}^{*}$. As $0<f_{1}<1$, one has

$$
\lim _{\mu_{00}^{*} \rightarrow 0} \frac{\mu_{00}^{*} P_{\mathrm{FA}}\left(\mu_{00}^{*}, \mu_{10}^{*}\right)}{1-f_{1}\left(\mu_{00}^{*}, \mu_{10}^{*}\right)}=0 .
$$

Thus for any fixed $\mu_{10}^{*}$, and for any decision threshold $0<$ $\gamma<1$, there exists exactly one $\mu_{00}^{*}$ satisfying (26).

Similarly, one may show that for any fixed $\mu_{00}^{*}$, and for any decision threshold $0<\gamma<1$, there exists only one $\mu_{10}^{*}$ satisfying (27).

Lemma 3. Let $G_{1}\left(\mu_{00}, \mu_{10}\right)=\left(\mu_{00}-\mu_{g}\right) \cdot f_{1}\left(\mu_{00}, \mu_{10}\right)$ and $G_{2}\left(\mu_{00}, \mu_{10}\right)=\left(\mu_{10}-\mu_{d}\right) \cdot f_{2}\left(\mu_{00}, \mu_{10}\right)$. Assume that

$$
\begin{array}{ll}
\frac{\partial G_{1}}{\partial \mu_{00}}>0, & \forall \mu_{00} \in\left[0, \mu_{g}\right], \mu_{10} \in\left[0, \mu_{d}\right], \\
\frac{\partial G_{2}}{\partial \mu_{10}}>0, & \forall \mu_{00} \in\left[0, \mu_{g}\right], \mu_{10} \in\left[0, \mu_{d}\right],
\end{array}
$$


and Conjectures 1 and 2 hold, then the equilibrium point $\left(\mu_{00}^{*}, \mu_{10}^{*}\right)$ is locally stable.

Proof: Consider a linearization of (24) around equilibrium with $\mu_{00}^{(\ell)}=\mu_{00}^{*}+\delta_{0}^{(\ell)}$, one gets

$$
\delta_{0}^{(\ell+1)}=\delta_{0}^{(\ell)} \cdot \frac{\partial F_{1}}{\partial \mu_{00}}\left(\mu_{00}^{*}, \mu_{10}^{*}\right),
$$

where $F_{1}\left(\mu_{00}, \mu_{10}\right)=\mu_{00} \cdot\left(f_{1}-P_{\mathrm{FA}}\right)+\mu_{\mathrm{g}} \cdot\left(1-f_{1}\right)$, with $0 \leq \mu_{00} \leq \mu_{\mathrm{g}}$ and $0 \leq \mu_{10} \leq \mu_{\mathrm{d}}$. Since $\frac{\partial F_{1}}{\partial \mu_{00}}\left(\mu_{00}^{*}, \mu_{10}^{*}\right)$ does not depend on $\ell$, the system (30) is locally stable around $\mu_{00}^{*}$ if

$$
\left|\frac{\partial F_{1}}{\partial \mu_{00}}\left(\mu_{00}^{*}, \mu_{10}^{*}\right)\right|<1 \text {. }
$$

Notice that

$$
\frac{\partial F_{1}}{\partial \mu_{00}}=\underbrace{f_{1}}_{<1}-\underbrace{\left(\mu_{\mathrm{g}}-\mu_{00}\right) \frac{\partial f_{1}}{\partial \mu_{00}}}_{>0}-\underbrace{\frac{\partial\left(\mu_{00} P_{\mathrm{FA}}\right)}{\partial \mu_{00}}}_{>0}<1
$$

holds since $\mu_{00} P_{\mathrm{FA}}$ has been assumed to be an increasing function of $\mu_{00}$. On the other hand, one needs $\frac{\partial F_{1}}{\partial \mu_{00}}>-1$, which leads to

$$
\begin{aligned}
& P_{\mathrm{FA}}\left(\mu_{00}, \mu_{10}\right)+\mu_{00} \frac{\partial P_{\mathrm{FA}}}{\partial \mu_{00}}+\left(\mu_{\mathrm{g}}-\mu_{00}\right) \frac{\partial f_{1}}{\partial \mu_{00}}-f_{1} \\
& =\frac{\partial\left(\mu_{00} \cdot P_{\mathrm{FA}}\right)}{\partial \mu_{00}}+\left(\mu_{\mathrm{g}}-\mu_{00}\right) \frac{\partial f_{1}}{\partial \mu_{00}}-f_{1} \\
& =\frac{\partial\left(\mu_{00} \cdot P_{\mathrm{FA}}\right)}{\partial \mu_{00}}-\frac{\partial G_{1}}{\partial \mu_{00}}<1
\end{aligned}
$$

One has assumed that $\mu_{00} P_{\mathrm{FA}}$ is concave, then

$$
\frac{\partial\left(\mu_{00} P_{\mathrm{FA}}\right)}{\partial \mu_{00}}<\lim _{\mu_{00} \rightarrow 0} \frac{\partial\left(\mu_{00} P_{\mathrm{FA}}\right)}{\partial \mu_{00}} \leq 1 .
$$

From (34) and (28), one obtains (33), thus (31) holds.

We can also prove that (27) is stable at $\mu_{10}^{*}$ in the similar way if Conjecture 1 and (29) hold. We conclude that the equilibrium point $\left(\mu_{00}^{*}, \mu_{10}^{*}\right)$ is locally stable if the conditions mentioned in Lemma 3 are satisfied.

\section{Simulation Results}

Consider a WSN of 1000 sensors randomly deployed according to a $2 \mathrm{D}$ PPP over a square of size $10 \times 10$ units, with $\mu=7$. To avoid boundary effects, only the sensors in the square of size $\left(10-2 R_{0}\right) \times\left(10-2 R_{0}\right)$ units are considered in the evaluations of $P_{\mathrm{D}}$ and $P_{\mathrm{FA}}$. In the outlier model presented in Section IV-A, $\eta=10$. All results have been averaged over 2000 realizations of the WSN.

First, Conjecture 1 and 2 are verified experimentally in Figure 3 and 4 respectively, for different values of parameters. The conditions in (28) and (29) are also verified numerically in Figure 5.

The performance of a single round of the non-adaptive DFD algorithm described in Section III-A is evaluated. Setting $\mu=7$, Figure 6 shows $P_{\mathrm{D}}$ as a function of $P_{\mathrm{FA}}$ for different values of the threshold $\gamma \in[0,1]$ and for various $\mu_{\mathrm{d}} / \mu \in\{0.02,0.05,0.1,0.2\}$.
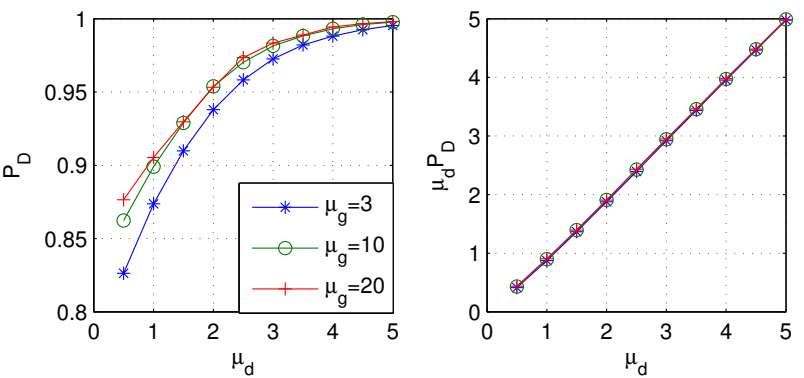

Fig. 3. Verification of Conjecture 1 , with $\mu_{\mathrm{g}} \in\{3,10,20\}$, and $\gamma=0.8$.
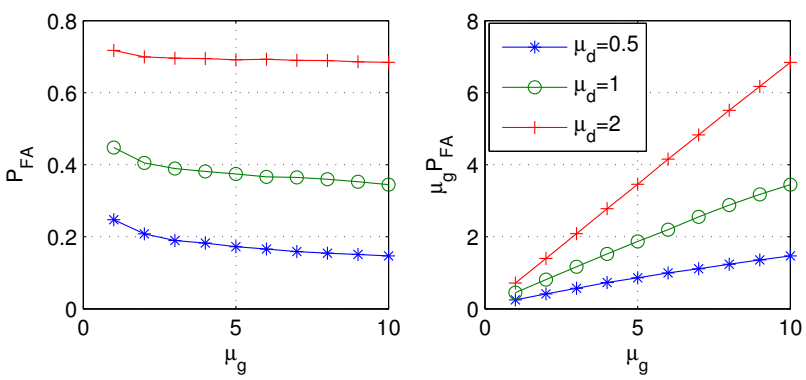

Fig. 4. Verification of Conjecture 2, with fixed $\mu_{\mathrm{d}} \in\{0.5,1,2\}$, and $\gamma=0.8$.
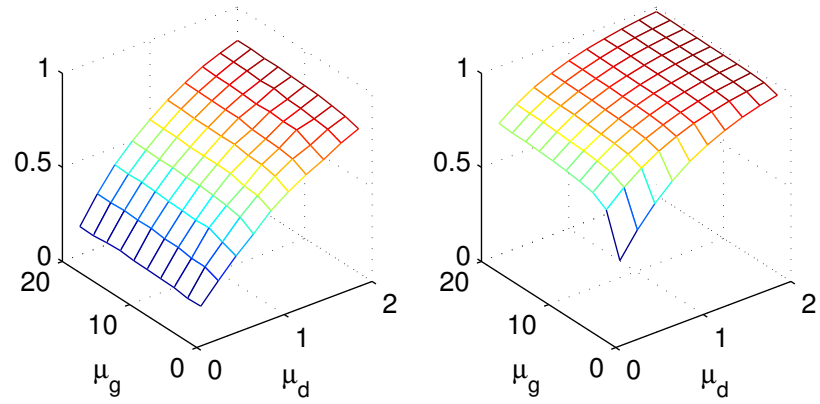

Fig. 5. Verification of $\frac{\partial G_{1}}{\partial \mu_{00}}>0$ (left) and $\frac{\partial G_{2}}{\partial \mu_{10}}>0$ (right) with $\mu_{\mathrm{g}}=20$ and $\mu_{\mathrm{d}}=2$.

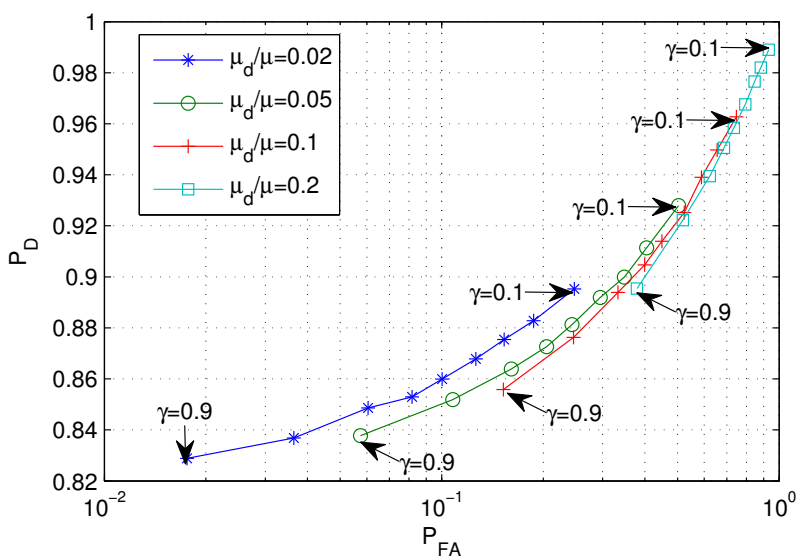

Fig. 6. $\quad P_{\mathrm{D}}$ as a function of $P_{\mathrm{FA}}$ for the non-adaptive DFD with different $\gamma$ and different densities of defective sensors; here $\mu=7$. 

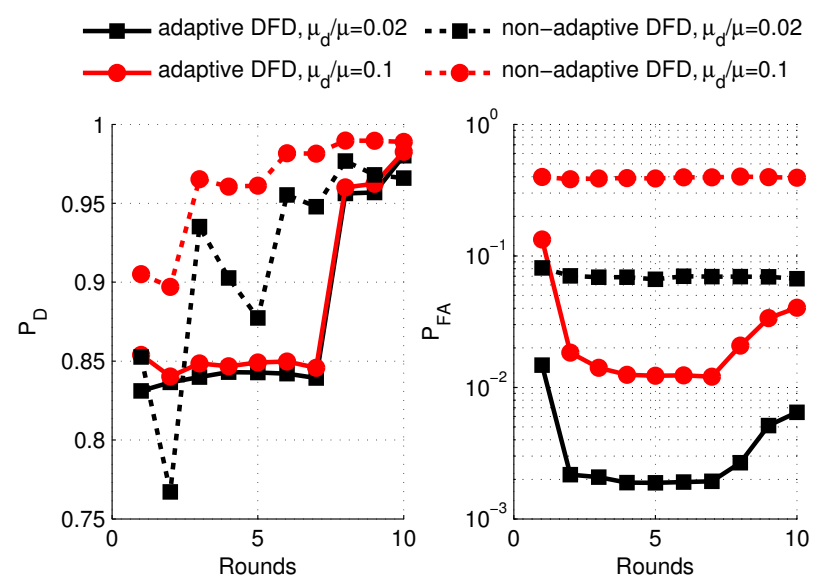

Fig. 7. $\quad P_{\mathrm{D}}$ (left) and $P_{\mathrm{FA}}$ (right) as a function of the number of rounds for the DFD algorithms, with $\mu=7$, and $\mu_{\mathrm{d}} / \mu \in\{0.02,0.1\}$

The roles played by the number of rounds on the achievable performance of both non-adaptive DFD and adaptive DFD are then investigated in Figure 7, which shows $P_{\mathrm{D}}$ and $P_{\mathrm{FA}}$ as a function of the round number $1 \leq \ell \leq L$, for $L=10$ and $\mu=7$. We set $\gamma=0.6$ in the non-adaptive DFD, As can be observed, $P_{\mathrm{D}}$ rapidly increases, whereas $P_{\mathrm{FA}}$ almost remains constant. In the case of adaptive DFD, we consider $L_{1}=7$, $L_{2}=3, \gamma_{1}=0.95$, and $\gamma_{2}=0.6$. Here $\gamma_{1}$ is chosen to be large to make the adaptive DFD easier to converge and to result in small $P_{\mathrm{FA}} . P_{\mathrm{FA}}$ can be reduced significantly during the first seven adaptive rounds. During the three following non-adaptive rounds, $P_{\mathrm{D}}$ improves rapidly, while $P_{\mathrm{FA}}$ remains small. This is due to the fact that most of the defective sensors have been detected and turned off during the previous adaptive phase, hence at the beginning of the non-adaptive rounds the residual $\mu_{d} / \mu$ is much lower than the initial $\mu_{d} / \mu$. Thus, the adaptive DFD algorithm performs better than the non-adaptive DFD algorithm: to achieve the similar $P_{\mathrm{D}}$, the resulting $P_{\mathrm{FA}}$ is much smaller by using the adaptive algorithm.

In Figure 8, the comparison between the proposed DFD and a reference DFD in [3] is reported in terms of $P_{\mathrm{D}}$ and $P_{\mathrm{FA}}$, after 10 rounds of the algorithm, in the case where $\mu=7$. A fair comparison between the two algorithms is quite difficult because they are based on different tests with non homogeneous parameters. In fact, in the reference DFD, the only parameter one can control is the threshold of a local test (similar to $\Delta$ in our case), whereas the performance of the proposed DFD depends on $\Delta, \gamma_{1}, \gamma_{2}, L_{1}$, and $L_{2}$. For example, we fix $\Delta=3 \sigma, L_{1}=7, L_{2}=3, \gamma_{1}=0.95$ and vary the value of $\gamma_{2}$. As can be seen in Figure 8, one may have better performance (with respect to $P_{\mathrm{FA}}$ and $P_{\mathrm{D}}$ ) if the parameters are properly chosen in the proposed DFD.

\section{CONCLUSIONS}

This work proposed two variants of a two-staged DFD algorithm, which allows each node to decide whether its sensor is producing outliers. The performance of the nonadaptive algorithm has been theoretically characterized. An analytical framework for the analysis of the adaptive variant has been sketched. The performance of both variants have been characterized by simulations, which enables to draw insights

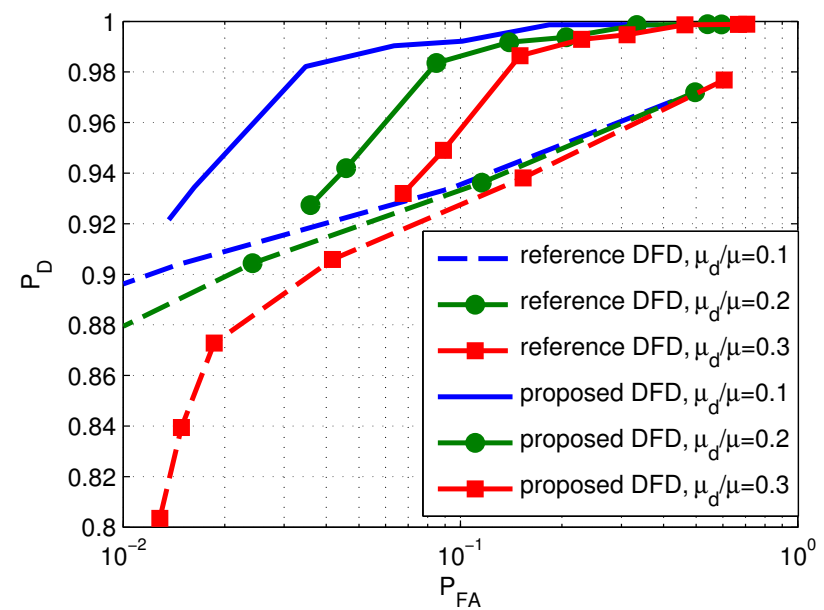

Fig. 8. $\quad P_{\mathrm{FA}}$ as a function of $P_{\mathrm{D}}$ for the reference DFD and the proposed DFD after 10 rounds, with $\mu_{d} / \mu \in\{0.1,0.2,0.3\}$.

on the impact of the algorithm parameters (number of rounds, local test threshold) and of the network topology (density of faulty sensors, size of the neighborhood) on the trade-off between $P_{\mathrm{D}}$ and $P_{\mathrm{FA}}$. The future work will be devoted to the study of other noise and outlier models and to a more formal verification of the conjectures.

\section{REFERENCES}

[1] R. Verdone, D. Dardari, G. Mazzini, and A. Conti, Wireless sensor and actuator networks: technologies, analysis and design. Academic Press, 2010.

[2] T. Quek, D. Dardari, and M. Win, "Energy efficiency of dense wireless sensor networks: To cooperate or not to cooperate," IEEE Journal on Selected Areas in Communications, vol. 25, no. 2, pp. 459-470, February 2007.

[3] J. Chen, S. Kher, and A. Somani, "Distributed fault detection of wireless sensor networks," in Proc Workshop DIWANS, New York, NY, USA, 2006, pp. $65-72$.

[4] M. Ding, D. Chen, K. Xing, and X. Cheng, "Localized fault-tolerant event boundary detection in sensor networks," in Proc IEEE INFOCOM, Miami, FL, USA, 2005, pp. $902-913$.

[5] J.-L. Gao, Y.-J. Xu, and X.-W. Li, "Weighted-median based distributed fault detection for wireless sensor networks," Journal of Software, vol. 18, no. 5, pp. 1208 - 1217, 2007.

[6] S. Ji, S.-F. Yuan, T.-H. Ma, and C. Tan, "Distributed fault detection for wireless sensor based on weighted average," in Proc NSWCTC, Wuhan, China, 2010, pp. $57-60$.

[7] M.-H. Lee and Y.-H. Choi, "Fault detection of wireless sensor networks," Computer Communications, vol. 31, no. 14, pp. 3469-3475, 2008.

[8] M. Cheraghchi, A. Hormati, A. Karbasi, and M. Vetterli, "Group testing with probabilistic tests: theory, design and application," IEEE Trans. Inf. Theory, vol. 57, no. 10, pp. 7057 - 7067, 2011.

[9] T. Tosic, N. Thomos, and P. Frossard, "Distributed sensor failure detection in sensor networks," Signal Processing, vol. 93, no. 2, pp. 399-410, 2013.

[10] M. Milanese, J. Norton, H. Piet-Lahanier, and E. Walter, Eds., Bounding Approaches to System Identification. New York, NY: Plenum Press, 1996.

[11] L. Jaulin, M. Kieffer, O. Didrit, and E. Walter, Applied Interval Analysis. London: Springer-Verlag, 2001.

[12] M. Haenggi, Stochastic geometry for wireless networks. Cambridge University Press, 2012. 